\title{
1 Editorial Prerogative and the Plant Genome
}

3 Daniel F. Voytas *

4

5 Department of Genetics, Cell Biology \& Development and Center for Genome

6 Engineering, University of Minnesota, Minneapolis, Minnesota 55455, USA

7

8 Corresponding author.voytas@umn.edu

9

10 Phone: +10 612 626-4509

11 Fax: +10 612 624-6264

12

13

14 Keywords: Genome editing; homologous recombination; non-homologous end-joining;

15 sequence-specific nucleases

16 
17 In the coming decades, plant agriculture must produce the food needed to sustain the

18 world's burgeoning population. This challenge is made more daunting in light of a

19 changing climate, scarcity of inputs such as water and fertilizer, and the need to grow

20 more crops on increasingly marginal land. To achieve food security, we will need to fully

21 exploit plant genetics to develop new crop varieties that produce higher yields of

22 healthier food. Crop improvement through breeding has been the foundation of modern

23 agriculture, resulting in steady increases in productivity of the major commodity crops

24 (Sleper and Poehlman, 2006). Additional genetic variation of value has been created

25 through mutagenesis strategies (Micke et al., 1990). More recently, gene addition

26 through transgenesis has provided valuable traits that impact yield, including herbicide

27 tolerance and resistance to insect pests (Gepts, 2002). Now, with the advent of genome

28 editing, we can directly alter the genetic blueprints of crop species - a powerful

29 approach to create genetic variation needed to produce abundant, healthy food.

$30 \quad$ Current approaches for editing plant genomes rely on introducing double strand

31 breaks in target loci; the manner in which the break is repaired achieves the desired

32 DNA sequence modification (Voytas, 2013). For many years, genome editing was

33 difficult to perform due to the lack of effective methods to produce nucleases that target

34 and cleave specific loci in complex genomes. The first programmable nucleases

35 required considerable protein engineering; for example, to alter the DNA sequence

36 specificity of rare-cutting endonucleases or zinc finger proteins (Cathomen and Joung,

37 2008; Arnould et al., 2011). The characterization of the DNA binding motif from TAL

38 effectors was an important advance for the field (Boch et al., 2009; Moscou and

39 Bogdanove, 2009). Variant TAL effector motifs in nature recognize each of the four

40 bases, and the motif is highly modular; that is, when arrays of motifs are assembled to

41 target novel DNA sequences, they recognize their intended targets $>90 \%$ of the time

42 (Cermak et al., 2011). More recently, the development of CRISPR/Cas reagents has 
43 irrevocably altered the field (Jinek et al., 2012; Cong et al., 2013; Mali et al., 2013).

44 Protein engineering is now largely obsolete, since targeting with CRISPR/Cas is

45 achieved simply through base-pairing between a guide RNA and the DNA target of

46 choice. Simplicity in reagent design and high activity of CRISPR/Cas nucleases make it

47 possible for the average molecular biologist to target DNA sequences with ease.

48 At present, editing one or a few genes can be performed with any of the available

49 nuclease platforms, as long as the reagent effectively binds and cleaves its intended

50 target site in vivo. For most scientists, particularly those in academia, the CRISPR/Cas

51 system is preferred, due to its effectiveness and simplicity. Companies with intellectual

52 property on the other nuclease platforms, however, will continue to use different

53 reagents to develop products such as new crop varieties. However, with academic

54 scientists largely unified around a single platform, improvements will be rapid. Already

55 available are Cas9 variants with improved target specificity, one of the initial concerns

56 about the system's efficacy (Kleinstiver et al., 2016; Slaymaker et al., 2016). Further,

57 CRISPR/Cas reagents have been developed that perform functions other than gene

58 editing, such as modulating transcription or altering the epigenetic status of a locus

59 (Thakore et al., 2016). Finally, since a single Cas nuclease can be directed to multiple

60 target sites by simply expressing multiple guide RNAs, it is straightforward to edit several

61 genes simultaneously (Cong et al., 2013).

62 Will other programmable nuclease platforms emerge? Most likely; however, it is

63 doubtful they will supplant CRISPR/Cas. For one, it is hard to imagine a simpler

64 targeting mechanism than base-pairing. Further, the rapid adoption of CRISPR/Cas

65 reagents for genetic analysis across diverse organisms, including plants, indicates they

66 serve the needs of most researchers; hence, there is little motivation to develop new

67 platforms. The field has moved from an era of reagent design to an era of reagent

68 deployment. 


\section{Editing genomes to increase biological understanding}

72 Before we can fully capitalize upon genome engineering for crop improvement, we must

73 have a sophisticated understanding of plant genomes and how they work. Already

74 available are high quality DNA sequences for genomes of dozens of important crop

75 plants; however, we really only understand the functional role of a fraction of plant

76 genes. The tools of genome engineering can help elucidate plant gene function.

77 Targeted mutagenesis makes it possible to connect the dots between genotype and 78 phenotype.

79 When double strand breaks are introduced in somatic cells, the broken

80 chromosomes are most typically rejoined through a mechanism called non-homologous

81 end-joining (NHEJ) (Puchta, 2005). NHEJ repair is sometimes imprecise, and,

82 consequently, the rejoined chromosome suffers a loss of nucleotides at the break site (or

83 less frequently, sustains a small insertion). If the insertion/deletion (indel) occurs within

84 a coding sequence, it can create a frameshift mutation, leading to gene inactivation.

85 Many reagents, including TALENs and CRISPR/Cas, create NHEJ-induced mutations at 86 remarkably high frequencies. For example, when protoplasts (plant cells lacking a cell

87 well) are transformed with constructs expressing CRISPR/Cas reagents, it is not

88 uncommon for $>25 \%$ of surveyed chromosomes to have mutations created by imprecise

89 NHEJ (Shan et al., 2013). For a handful of plant species, mutagenized protoplasts can

90 be regenerated into plants, making it possible to assess the phenotypic consequence of

91 NHEJ-induced mutations at the whole plant level (Clasen et al., 2016).

92 Only a handful of plant species, however, can be regenerated from protoplasts,

93 and so mutations in most plants are created by first generating a transgenic plant that

94 expresses the sequence-specific nuclease and a selectable marker gene (Voytas, 
95 2013). As the transgenic plant grows, cells are mutagenized, including those in the

96 meristem that ultimately produce flowers and seed. Mutagenesis, therefore, occurs in

97 planta, and heritable mutations can be reliably obtained in many species by this strategy

98 (Weeks et al., 2016). For some plants, such as Arabidopsis, mutagenesis frequencies

99 have been increased by using promoters that express the nuclease preferentially in the

100 germline (Wang et al., 2015; Mao et al., 2016). An advantage of in planta strategies is

101 that they use standard transformation methods, which are available for diverse plants.

102 Since it is unlikely that the targeted mutation and the nuclease-encoding transgene are

103 genetically linked, the latter can be segregated away through crossing, leaving a plant

104 with only the desired mutation.

105 The efficiency of sequence-specific nucleases for in planta mutagenesis is

106 striking. For example, six homeoalleles in wheat were simultaneously knocked out,

107 resulting in a fungus-resistant cultivar (Wang et al., 2014). Similarly, eight genes were

108 simultaneously mutated in Arabidopsis (Ma et al., 2015). Although resource intensive, it

109 is not unrealistic to contemplate creating a mutant plant library for species such as rice,

110 in which each member of the library is a plant with a different gene inactivated. Further,

111 the extensive collections of T-DNA insertion mutants in plants like Arabidopsis could be

112 augmented through targeted mutagenesis to create a complete knockout library for this

113 model species. For scientists working in baker's yeast, the completion of a knockout

114 collection proved invaluable for dissecting gene function (Giaever et al., 2002). One of

115 the most important outcomes of our newfound editorial control over plant genomes will

116 be the wealth of basic biological information we glean regarding plant gene function.

117 Clearly targeted mutagenesis can help advance basic biology. However, there

118 are also some traits of value that can be created through gene inactivation, such as

119 removal of anti-nutritionals or toxins (e.g. trypsin inhibitors in soybean, ricin in caster oil).

120 Further, metabolic pathways can be blocked at specific steps to accumulate desirable 
121 precursors. This has been done in soybean by knocking out a seed-expressed fatty acid

122 desaturase, thereby lowering the production of downstream products, namely

123 polyunsaturated fats, and enriching for healthier precursors, namely monounsaturated

124 fats (Haun et al., 2014). Plants with traits enabled by targeted mutagenesis are likely to

125 soon enter the food supply. In the US, the USDA has ruled that in several cases,

126 transgene free, NHEJ-induced mutants are not regulated (Jones, 2015). This sets an

127 important precedent, indicating that mutations created with sequence-specific nucleases

128 will be treated in the same way as mutations created by chemical mutagens or ionizing

129 radiation. Plants with targeted mutations can therefore quickly be tested in the field; field

130 trials provide the most rigorous assessment of a trait's value.

131

\section{Editing plant genomes with finesse}

134 Whereas targeted mutagenesis with sequence-specific nucleases has worked well in

135 many species and is beginning to facilitate a greater understanding of gene function,

136 sequence modifications other than NHEJ-induced indels are often desired. For

137 example, specific nucleotide substitutions, which alter key amino acid residues in a

138 protein or change promoter function, might be of value. Such precise modifications

139 allow more sophisticated tests of gene function, and by extension, agronomically

140 relevant genetic variation can be moved into the genomes of crop species to introduce

141 traits of value.

142 Precision genome editing is best enabled by harnessing the other mechanism by

143 which broken chromosomes are repaired, namely homologous recombination (HR). In

$144 \mathrm{HR}$, a template is provided with the desired sequence alterations to be incorporated into

145 the genome (Puchta, 2005). Sequence alterations on the template are copied into the

146 break site, thereby altering the locus. The types of sequence modifications that can be 
147 introduced through HR are expansive, ranging from single nucleotide substitutions to the

148 insertion of one or more transgenes. A challenge in achieving HR, however, is the need

149 to simultaneously deliver the template and nuclease. Further, HR is inefficient in

150 somatic cells, and the competing pathway - NHEJ - predominates (Puchta, 2005).

151 Because of these challenges, remarkably few examples of HR-mediated genome editing

152 have been reported in plants.

153 For the few plants species that can be regenerated from protoplasts, direct

154 delivery of the nuclease and donor can achieve HR at reasonable efficiencies - in the

155 range of $1 \%$ of transformed cells (Townsend et al., 2009). Populations of edited cells

156 can be regenerated into plants and screened to identify those with the desired DNA

157 sequence modification. For other plant species, in which differentiated tissues are

158 transformed by Agrobacterium or biolistics, recovering edited plants is a bit of a numbers

159 game. Only subsets of cells in the tissues are transformed, and of those, only a fraction

160 undergoes HR. Donor templates that incorporate selectable markers at the target locus

161 can help identify cells having sustained HR (Sun et al., 2007). The downside is that the

162 edited plant contains the selectable marker gene, which may be undesirable for product

163 development. Further, if the goal is precise genome editing, then incorporating a marker

164 gene certainly lacks the sought-after precision.

165 Similar to the strategies described above for NHEJ-induced mutagenesis, in

166 planta strategies have also been used for HR-based targeted modification (Fauser et al.,

167 2012). In this approach, a nuclease cassette and the repair template are integrated into

168 the genome at a random chromosomal site. The template is flanked by nuclease cut

169 sites, such that when the intended genomic target site is cut, the template is released by

170 the nuclease, enabling it to move throughout the nucleus and recombine with the target.

171 Other strategies to improve gene targeting have involved developing vectors to improve

172 delivery of the nuclease and donor template. For example, geminivirus-based DNA 
173 replicons amplify to high copy number in plant cells (Baltes et al., 2014; Cermak et al.,

174 2015). Thus, the replicons produce an abundance of nuclease and repair template,

175 often increasing HR frequencies an order of magnitude or more. Finally, it is also

176 possible to knockout function of key enzymes in the NHEJ pathway, thereby increasing

177 the frequency with which broken chromosomes are repaired through HR (Qi et al.,

178 2013).

179 Despite these promising approaches, editing plant genomes with precision

180 remains difficult. With so much interest in genome engineering among plant biologists,

181 and with the availability of powerful easy-to-use reagents to make targeted chromosome

182 breaks, it is likely that the field will witness considerable technical improvements in the

183 coming years. Such improvements should soon make it easy and routine to perform

184 plant genome editing with finesse.

185

186 Conclusions

187

188 The advent first of TALENs and then CRISPR/Cas reagents made it possible for many

189 plant scientists to begin modifying plant genomes in sophisticated ways. NHEJ-induced

190 targeted mutagenesis is now efficient in many plant species and promises to be a

191 powerful approach for elucidating plant gene function. Efficient methods to precisely edit

192 plant genomes through HR are the next challenge; however, improvements are likely to

193 be rapid. Genetic variation created through genome editing will shortly enter the food

194 supply, and it is the hope that traits created through genome editing will help meet the

195 world's increasing need for more and healthier food.

196

197 


\section{Acknowledgements}

199 This work was supported in part by grants from the National Science Foundation (IOS200 1444511and IOS-1339209).

202 References

203 Arnould, S., Delenda, C., Grizot, S., Desseaux, C., Paques, F., Silva, G.H., Smith, J., 2011. The I-Crel meganuclease and its engineered derivatives: applications from cell modification to gene therapy. Protein Eng. Des. Sel. 24, 27-31.

Baltes, N.J., Gil-Humanes, J., Cermak, T., Atkins, P.A., Voytas, D.F., 2014. DNA replicons for plant genome engineering. Plant Cell 26, 151-163.

Boch, J., Scholze, H., Schornack, S., Landgraf, A., Hahn, S., Kay, S., Lahaye, T., Nickstadt, A., Bonas, U., 2009. Breaking the code of DNA binding specificity of TAL-type III effectors. Science 326, 1509-1512.

211 Cathomen, T., Joung, J.K., 2008. Zinc-finger nucleases: the next generation emerges.

$212 \quad$ Mol. Ther. 16, 1200-1207.

213 Cermak, T., Baltes, N.J., Cegan, R., Zhang, Y., Voytas, D.F., 2015. High-frequency, 214 precise modification of the tomato genome. Genome Biol. 16, 232.

215 Cermak, T., Doyle, E.L., Christian, M., Wang, L., Zhang, Y., Schmidt, C., Baller, J.A., 216 Somia, N.V., Bogdanove, A.J., Voytas, D.F., 2011. Efficient design and assembly 217 of custom TALEN and other TAL effector-based constructs for DNA targeting. $218 \quad$ Nucleic Acids Res. 39, e82.

219 Clasen, B.M., Stoddard, T.J., Luo, S., Demorest, Z.L., Li, J., Cedrone, F., Tibebu, R., 220 Davison, S., Ray, E.E., Daulhac, A., Coffman, A., Yabandith, A., Retterath, A., 221 Haun, W., Baltes, N.J., Mathis, L., Voytas, D.F., Zhang, F., 2016. Improving cold 222 storage and processing traits in potato through targeted gene knockout. Plant 223 Biotechnol. J. 14, 169-176. 
224 Cong, L., Ran, F.A., Cox, D., Lin, S., Barretto, R., Habib, N., Hsu, P.D., Wu, X., Jiang,

225 W., Marraffini, L.A., Zhang, F., 2013. Multiplex genome engineering using

226 CRISPR/Cas systems. Science 339, 819-823.

227 Fauser, F., Roth, N., Pacher, M., Ilg, G., Sanchez-Fernandez, R., Biesgen, C., Puchta, 228 H., 2012. In planta gene targeting. Proc. Natl. Acad. Sci. USA 109, 7535-7540.

229 Gepts, P., 2002. A comparison between crop domestication, classical breeding, and 230 genetic engineering. Crop Sci. 42, 1780-1790.

231 Giaever, G., Chu, A.M., Ni, L., Connelly, C., Riles, L., Veronneau, S., Dow, S., LucauDanila, A., Anderson, K., Andre, B., Arkin, A.P., Astromoff, A., El-Bakkoury, M., Bangham, R., Benito, R., Brachat, S., Campanaro, S., Curtiss, M., Davis, K., Deutschbauer, A., Entian, K.D., Flaherty, P., Foury, F., Garfinkel, D.J., Gerstein, M., Gotte, D., Guldener, U., Hegemann, J.H., Hempel, S., Herman, Z., Jaramillo, D.F., Kelly, D.E., Kelly, S.L., Kotter, P., LaBonte, D., Lamb, D.C., Lan, N., Liang, H., Liao, H., Liu, L., Luo, C., Lussier, M., Mao, R., Menard, P., Ooi, S.L., Revuelta, J.L., Roberts, C.J., Rose, M., Ross-Macdonald, P., Scherens, B., Schimmack, G., Shafer, B., Shoemaker, D.D., Sookhai-Mahadeo, S., Storms, R.K., Strathern, J.N., Valle, G., Voet, M., Volckaert, G., Wang, C.Y., Ward, T.R.,

245 Haun, W., Coffman, A., Clasen, B.M., Demorest, Z.L., Lowy, A., Ray, E., Retterath, A., 246 Stoddard, T., Juillerat, A., Cedrone, F., Mathis, L., Voytas, D.F., Zhang, F., 2014. 247 Improved soybean oil quality by targeted mutagenesis of the fatty acid 248 desaturase 2 gene family. Plant Biotechnol. J. 12, 934-940. 
249 Jinek, M., Chylinski, K., Fonfara, I., Hauer, M., Doudna, J.A., Charpentier, E., 2012. A

250 programmable dual-RNA-guided DNA endonuclease in adaptive bacterial

251 immunity. Science 337, 816-821.

252 Jones, H.D., 2015. Regulatory uncertainty over genome editing. Nat Plants 1, 1-3.

253 Kleinstiver, B.P., Pattanayak, V., Prew, M.S., Tsai, S.Q., Nguyen, N.T., Zheng, Z.,

254 Joung, J.K., 2016. High-fidelity CRISPR-Cas9 nucleases with no detectable

255 genome-wide off-target effects. Nature 529, 490-495.

256 Ma, X., Zhang, Q., Zhu, Q., Liu, W., Chen, Y., Qiu, R., Wang, B., Yang, Z., Li, H., Lin, Y.,

257 Xie, Y., Shen, R., Chen, S., Wang, Z., Chen, Y., Guo, J., Chen, L., Zhao, X.,

258 Dong, Z., Liu, Y.G., 2015. A robust CRISPR/Cas9 system for convenient, high-

259 efficiency multiplex genome editing in monocot and dicot plants. Mol. Plant 8, $260 \quad 1274-1284$.

261 Mali, P., Yang, L., Esvelt, K.M., Aach, J., Guell, M., DiCarlo, J.E., Norville, J.E., Church,

262 G.M., 2013. RNA-guided human genome engineering via Cas9. Science 339, 263 823-826.

264 Mao, Y., Zhang, Z., Feng, Z., Wei, P., Zhang, H., Botella, J.R., Zhu, J.K., 2016.

265 Development of germ-line-specific CRISPR-Cas9 systems to improve the

266 production of heritable gene modifications in Arabidopsis. Plant Biotechnol. J. 14, $267 \quad 519-532$.

268 Micke, A., Donini, B., Maluszynski, M., 1990. Induced mutations for crop improvement,

269 Mutation Breeding Review, FAO/IAEA. International Atomic Energy Agency,

$270 \quad$ Vienna, Austria.

271 Moscou, M.J., Bogdanove, A.J., 2009. A simple cipher governs DNA recognition by TAL 272 effectors. Science 326, 1501.

273 Puchta, H., 2005. The repair of double-strand breaks in plants: mechanisms and 274 consequences for genome evolution. J. Exp. Bot. 56, 1-14. 
275 Qi, Y., Zhang, Y., Zhang, F., Baller, J.A., Cleland, S.C., Ryu, Y., Starker, C.G., Voytas,

276 D.F., 2013. Increasing frequencies of site-specific mutagenesis and gene

277 targeting in Arabidopsis by manipulating DNA repair pathways. Genome Res. 23, $278 \quad 547-554$.

279 Shan, Q., Wang, Y., Li, J., Zhang, Y., Chen, K., Liang, Z., Zhang, K., Liu, J., Xi, J.J., Qiu, 280 J.L., Gao, C., 2013. Targeted genome modification of crop plants using a 281 CRISPR-Cas system. Nat. Biotechnol. 31, 686-688.

282 Slaymaker, I.M., Gao, L., Zetsche, B., Scott, D.A., Yan, W.X., Zhang, F., 2016.

283 Rationally engineered Cas9 nucleases with improved specificity. Science 351, $284 \quad 84-88$.

285 Sleper, D.A., Poehlman, J.M., 2006. Breeding Field Crops, 5th Ed. ed. Blackwell 286 Publishing.

287 Sun, Y., Thompson, M., Lin, G., Butler, H., Gao, Z., Thornburgh, S., Yau, K., Smith, 288 D.A., Shukla, V.K., 2007. Inositol 1,3,4,5,6-pentakisphosphate 2-kinase from 289 maize: molecular and biochemical characterization. Plant Physiol. 144, 1278$290 \quad 1291$.

291 Thakore, P.I., Black, J.B., Hilton, I.B., Gersbach, C.A., 2016. Editing the epigenome: 292 technologies for programmable transcription and epigenetic modulation. Nat. $293 \quad$ Methods 13, 127-137.

294 Townsend, J.A., Wright, D.A., Winfrey, R.J., Fu, F., Maeder, M.L., Joung, J.K., Voytas, 295 D.F., 2009. High-frequency modification of plant genes using engineered zinc296 finger nucleases. Nature 459, 442-445.

297 Voytas, D.F., 2013. Plant genome engineering with sequence-specific nucleases. Annu. 298 Rev. Plant Biol. 64, 327-350. 
299 Wang, Y., Cheng, X., Shan, Q., Zhang, Y., Liu, J., Gao, C., Qiu, J.L., 2014.

300 Simultaneous editing of three homoeoalleles in hexaploid bread wheat confers

301 heritable resistance to powdery mildew. Nat. Biotechnol. 32, 947-951.

302 Wang, Z.P., Xing, H.L., Dong, L., Zhang, H.Y., Han, C.Y., Wang, X.C., Chen, Q.J., 2015.

303 Egg cell-specific promoter-controlled CRISPR/Cas9 efficiently generates

304 homozygous mutants for multiple target genes in Arabidopsis in a single

305 generation. Genome Biol. 16, 144.

306 Weeks, D.P., Spalding, M.H., Yang, B., 2016. Use of designer nucleases for targeted

307 gene and genome editing in plants. Plant Biotechnol. J. 14, 483-495.

308 